MODELING, IDENTIFICATION AND CONTROL, 1996, VOL. 17, NO. 2, 121-133

doi:10.4173/mic.1996.2.5

\title{
Passive output feedback and observer based autopilots: a comparative study
}

\author{
MARIT J. PAULSEN† and OLAV EGELAND +
}

Keywords: Comparative study, ship autopilots, passive controllers, observer based controllers, output feedback controllers

Two methods for course-keeping of a ship are studied where no measurements of the yaw rate are available. The two methods are a passive output feedback controller and a controller-observer structure. A comparison with special attention to stability and wave filtering properties, is provided. Finally, a case study of a ship autopilot is included.

\section{Introduction}

In course-keeping for marine vehicles, one of the main problems is the influence from wave disturbances. To avoid unnecessary use and wear of actuators we do not want to compensate for first order wave disturbances. Therefore, wave filtering is introduced. Two strategies for wave filtering have been widely used. The conventional approach is to use a notch or dead-band filter in the control loop to attenuate the influence from wave disturbances on the control input, see for instance Sargent and Cowgill (1976). Another method is to design an observer to estimate the high frequency and low frequency component of the output and use the low frequency estimate for feedback control. The latter method was introduced by Balchen et al. (1976). Later, several papers on this method have been published, e.g. Balchen et al. (1980), Grimble et al. (1980b), and Sælid et al. (1983b). For control purposes a PD or PID controller is most widely used. These controller-observer structures will be referred to as observer based controllers throughout this paper.

In Paulsen et al. (1994) a passive output feedback controller with wave filtering for marine vehicles is presented. The design is inspired by the full state feedback system for dynamic positioning presented by Johannessen and Egeland (1993), which in Schei (1995) is shown to be similar to a notch filter with PD-controller. However, there is a relation between the parameters in the passive controller that ensures the passivity properties of the control system. In Paulsen et al. (1994), the passive dynamic positioning system is extended to the case with no velocity measurements.

The passive output feedback controller has certain similarities with the observer based controller, and it is interesting to investigate the performance of the two controllers in particular with respect to wave filtering and stability properties. In this paper such a comparison is presented.

Received 20 August 1995

$\dagger$ Norwegian Institute of Technology, N-7034 Trondheim, Norway. E-mail: Marit.Paulsen@itk.unit.no, Olav.Egeland@itk.unit.no

Presented at the 3rd IFAC Workshop on Control Applications in Marine Systems, Trondheim, Norway, May 1995 (CAMS '95). 


\section{Ship and wave models}

Consider the linear model for the low frequency yaw motion of a ship by Nomoto et al. (1957)

$$
m^{*} \ddot{\psi}_{L}(t)+d^{*} \dot{\psi}_{L}(t)=\delta(t)
$$

where $m^{*}$ and $d^{*}$ are positive constants, $\psi_{L}(t)$ is the yaw angle, and $\delta(t)$ is the control input, that is, the rudder angle. In the frequency domain the ship model is described by

$$
\psi_{L}(s)=\frac{1}{s\left(m^{*} s+d^{*}\right)} \delta(s)=\frac{1}{h_{S}(s)} \delta(s)
$$

For the analysis of the stability properties of the total system later in this paper, the passivity properties of the ship are investigated. Using standard notation (Desoer and Vidyasagar 1975), we find that

$$
\begin{aligned}
\left\langle\delta, \dot{\psi}_{L}\right\rangle_{T} & =\left\langle m^{*} \ddot{\psi}_{L}+d^{*} \dot{\psi}_{L}, \dot{\psi}_{L}\right\rangle_{T} \\
& =\left\langle d^{*} \dot{\psi}_{L}, \dot{\psi}_{L}\right\rangle_{T}+\int_{0}^{T} m^{*} \ddot{\psi}_{L} \dot{\psi}_{L} \mathrm{~d} t \\
& \geqslant d^{*}\left\|\dot{\psi}_{L}\right\|_{2 T}^{2}+\beta
\end{aligned}
$$

where

$$
\beta=-\frac{1}{2} m * \dot{\psi}_{L}(0)^{2}
$$

Thus, the system with input $\delta(t)$ and output $\psi_{L}(t)$ is output strictly passive as defined by Hill and Moylan (1977). It follows that

$$
\left|<\frac{j \omega}{h_{S}(j \omega)}\right|<90^{\circ}
$$

This means that the phase shift of the transfer function from $\delta$ to $\psi_{L}$ is less than $90^{\circ}$.

In this paper we assume that the only disturbances acting on the ship are first order wave disturbances. Compensation of higher order wave disturbances and constant current and wind disturbances is not discussed. Integral action can be included in the controllers to compensate for the constant disturbances.

As in Balchen et al. (1976) the wave disturbance is considered to be an output disturbance. This means that the measured output $\psi(t)$ is

$$
\psi(t)=\psi_{L}(t)+\psi_{H}(t)
$$

where $\psi_{L}(t)$ was defined in (1). The signal $\psi_{H}(t)$ can be thought of as the yaw angle induced by the wave disturbances. Several models for describing this high frequency motion of the ship exist. In Fossen (1994) an overview of some models is provided. For the simulation study, Section 7, we choose to implement a linear approximation to the nonlinear wave models by Grimble et al. (1980a). The transfer function is given as

$$
\begin{aligned}
\psi_{H}(s) & =h_{W}(s) \eta(s) \\
& =\frac{K_{w} s^{2}}{\left(s^{2}+2 \zeta \omega_{n} s+\omega_{n}^{2}\right)^{2}} \eta(s)
\end{aligned}
$$


$\eta$ is a normally distributed random variable with zero mean value and variance equal to one, $\omega_{n}$ is the dominating wave frequency, $\zeta$ is the relative damping ratio of the waves, and $K_{w}$ is a gain that is dependent on the wave height.

\section{Passive output feedback controller}

In this section the passive output feedback controller proposed in Paulsen et al. (1994) is briefly reviewed for use in the comparison. The control law is

$$
\delta=k_{P 0}\left(\psi_{0}-\psi\right)
$$

where $k_{P 0}$ is a positive regulator constant, $\psi(t)$ is the measured yaw angle, and $\psi_{0}(t)$ is a virtual reference trajectory given by

$$
k_{P 0}\left(\psi_{0}-\psi\right)=k_{P 1}\left(\psi_{1}-\psi_{0}\right)+k_{D 1}\left(\dot{\psi}_{1}-\dot{\psi}_{0}\right)
$$

where $\psi_{i}(t), i \in\{1, \ldots, n\}$, are given by the $n$ equations

$$
\begin{gathered}
m_{1} \ddot{\psi}_{1}+k_{P 1}\left(\psi_{1}-\psi_{0}\right)+k_{D 1}\left(\dot{\psi}_{1}-\dot{\psi}_{0}\right)+k_{P 2}\left(\psi_{1}-\psi_{2}\right)+k_{D 2}\left(\dot{\psi}_{1}-\dot{\psi}_{2}\right)=0 \\
\vdots \\
m_{i} \ddot{\psi}_{i}+k_{P i}\left(\psi_{i}-\psi_{i-1}\right)+k_{D i}\left(\dot{\psi}_{i}-\dot{\psi}_{i-1}\right)+k_{P(i+1)}\left(\psi_{i}-\psi_{i+1}\right)+k_{D(i+1)}\left(\dot{\psi}_{i}-\dot{\psi}_{i+1}\right)=0 \\
\vdots \\
m_{v} \ddot{\psi}_{n}+k_{P n}\left(\psi_{n}-\psi_{n-1}\right)+k_{D n}\left(\dot{\psi}_{n}-\dot{\psi}_{n-1}\right)+k_{P(n+1)} \psi_{n}+k_{D(n+1)} \dot{\psi}_{n}=0
\end{gathered}
$$

Here and throughout the paper, the desired yaw angle is equal to zero. The regulator parameters $m_{i}, i \in\{1, \ldots, n\}, k_{P i}, i \in\{1, \ldots, n+1\}$, and $k_{D i}, i \in\{1, \ldots, n+1\}$, are positive and constant.

Figure 1 shows how the control system can be interpreted as a mechanical system consisting of $n$ masses, $n+1$ dampers, and $n+2$ springs. For simplicity, the controller with only one virtual mass $(n=1)$ will be considered in the following analysis.

For the further analysis it is interesting to determine the transfer function from $\psi$ to $\delta$. Laplace transformation of (7)-(9), $n=1$, gives

$$
\begin{aligned}
\delta(s) & =k_{P 0}\left(\psi_{0}(s)-\psi(s)\right) \\
k_{P 0}\left(\psi(s)-\psi_{0}(s)\right) & =h_{1}(s)\left(\psi_{0}(s)-\psi_{1}(s)\right) \\
h_{2}(s) \psi_{1}(s) & =h_{1}(s)\left(\psi_{0}(s)-\psi_{1}(s)\right)
\end{aligned}
$$

where $h_{1}(s)$ and $h_{2}(s)$ are defined as:

$$
\begin{aligned}
& h_{1}(s)=k_{D 1} s+k_{P 1} \\
& h_{2}(s)=m_{1} s^{2}+k_{D 2} s+k_{P 2}
\end{aligned}
$$



Figure 1. Mechanical interpretation of passive output feedback controller. 
Further calculations yield

$$
\begin{aligned}
\frac{\delta}{\psi}(s) & =h_{R 1}(s) \\
& =\frac{-k_{P 0} h_{1}(s) h_{2}(s)}{h_{1}(s) h_{2}(s)+k_{P 0}\left(h_{1}(s)+h_{2}(s)\right)}
\end{aligned}
$$

Note that the order of both the numerator and denominator is three. Thus, the relative degree of the transfer function $h_{R 1}(s)$ is zero. The passive controller $h_{R 1}$ has almost the same structure as a notch filter with a low pass filter and PD-controller. The low pass filter is due to the estimation of the velocity.

\section{Observer based wave filtering and control}

In this section we present the observer based controller, see for instance Sælid et al. (1983b). By observer based, we refer to that the high frequency and low frequency motion of the ship are estimated by using models of the ship and the first order wave disturbance. The low frequency estimate is used for feedback control and given in the time domain as

$$
\begin{aligned}
& \dot{\hat{\psi}}_{L}=\hat{r}_{L}+k_{1} \bar{\psi} \\
& \dot{\hat{r}}_{L}=\frac{1}{m}\left(-d \hat{r}_{L}+\delta\right)+k_{2} \bar{\psi}
\end{aligned}
$$

and the high frequency estimate is given as

$$
\begin{aligned}
& \dot{\hat{\psi}}_{H}=\hat{r}_{H}+k_{3} \bar{\psi} \\
& \dot{\hat{r}}_{H}=-2 \zeta_{0} \omega_{n} \hat{r}_{H}-\omega_{n}^{2} \hat{\psi}_{H}+k_{4} \bar{\psi}
\end{aligned}
$$

Here, $\hat{\psi}_{L}(t)$ and $\hat{\mathbf{r}}_{L}(t)$ are the low frequency estimate of the yaw angle and yaw rate, respectively. Similarly, $\hat{\psi}_{H}(t)$ and $\hat{r}_{H}(t)$ are the estimates of the high frequency motion. The constant parameters $m$ and $d$ are chosen equal to or as close as possible to the ship's actual parameters, $m^{*}$ and $d^{*}$. The parameter $\zeta_{0}$ is a relative damping coefficient which should be chosen small to give a strong notch effect. It should not necessarily be chosen equal to the parameter $\zeta$ in the wave disturbance model (6). Further, $\bar{\psi}(t)$ is defined by

$$
\bar{\psi}(t)=\psi(t)-\hat{\psi}_{L}(t)-\hat{\psi}_{H}(t)
$$

The parameters $k_{i}, i \in\{1, \ldots, 4\}$ must be chosen such that the total system is stable. This can be done in several ways. An optimal estimation technique known as Kalman filtering is used by Balchen et al. (1976), Balchen et al. (1980), Grimble et al. (1980b), and Sælid et al. (1983b) among others. The parameters may also be chosen by for instance pole placement or similar techniques. In this paper, we assume that the observer has constant parameters.

Laplace transformation of (12)-(13) yields

$$
\begin{aligned}
\hat{\psi}_{L}(s) & =\frac{k_{1} m s+k_{1} d+k_{2} m}{(m s+d) s} \bar{\psi}(s)+\frac{1}{(m s+d) s} \delta(s) \\
& =\frac{k_{L}(s)}{h_{L}(s)} \bar{\psi}(s)+\frac{1}{h_{L}(s)} \delta(s)
\end{aligned}
$$




$$
\begin{aligned}
\hat{\psi}_{H}(s) & =\frac{k_{3} s+k_{4}+k_{3} 2 \zeta_{0} \omega_{n}}{s^{2}+2 \zeta_{0} \omega_{n} s+\omega_{n}^{2}} \bar{\psi}(s) \\
& =\frac{k_{H}(s)}{h_{H}(s)} \bar{\psi}(s)
\end{aligned}
$$

It seems reasonable to choose $k_{4}=-k_{3} 2 \zeta_{0} \omega_{n}$ (Sælid et al. 1983b), since no low frequency components will then be present in the high frequency model (15). Thus, we assume $k_{4}=-k_{3} 2 \zeta_{0} \omega_{n}$ in the rest of the paper.

We choose a PD-controller

$$
\delta(s)=-\left(k_{D} s+k_{P}\right) \hat{\psi}_{L}(s)=-h_{P D}(s) \hat{\psi}_{L}(s)
$$

for comparison with the controller in Section 3. The control parameters may be chosen by minimizing a weighting criterion (optimal control). Combining a Kalman filter for estimation with an optimal controller is referred to as Linear Quadratic Gaussian (LQG) control. The transfer function from $\psi$ to $\delta$ becomes

$$
\frac{\delta}{\psi}(s)=h_{R 2}(s)=\frac{-h_{P D} k_{L} h_{H}}{\left(h_{L}+h_{P D}\right)\left(h_{H}+k_{H}\right)+k_{L} h_{H}}
$$

The order of the numerator and denominator is four. This means that the relative degree of this controller is also zero. Previously, Grimble (1978) has shown that a special case of the observer based filter, that is the Kalman filter, is approximately equal to a notch filter with a low pass filter in cascade.

\section{Wave filtering properties}

We will now give an analysis of the wave filtering properties of the two controllers. We are particularly interested in how the input $\delta$ is affected by $\psi_{H}$, which is considered to be a wave disturbance acting on the output. To this end, we will compare the transfer functions from $\psi_{H}$ to $\delta$. First, we rewrite the controller transfer functions according to

$$
h_{R i}(s)=\frac{n_{R i}(s)}{d_{R i}(s)}
$$

where $n_{R i}$ and $d_{R i}, i \in\{1,2\}$, denote the numerator and denominator, respectively, of the controllers defined in (11) and (17). The transfer functions $\delta / \psi_{H}$ for both controllers become

$$
\frac{\delta}{\psi_{H}}(s)=\frac{n_{R i}(s) h_{S}(s)}{d_{R i}(s) h_{S}(s)-n_{R i}(s)}
$$

where $h_{S}(s)$ is defined in (2). The second order term $m_{1} s^{2}+k_{D 2} s+k_{P 2}$ in $n_{R 1}$ and $s^{2}+2 \zeta_{0} \omega_{n} s+\omega_{n}^{2}$ in $n_{R 2}$ give the main contribution to the wave filtering. The second order term in $n_{R 1}$ can be rewritten as $m_{1}\left(s^{2}+2 \zeta_{p} \omega_{n} s+\omega_{n}^{2}\right)$, which implies that the controller constants $k_{D 2}$ and $k_{P 2}$ should be chosen as

$$
k_{D 2}=2 \zeta_{p} \omega_{n} m_{1}, \quad k_{P 2}=\omega_{n}^{2} m_{1} .
$$

If the relative damping coefficients $\zeta_{0}$ and $\zeta_{p}$ are chosen less than 1 , the second order terms will have complex conjugated zeros, and notch effect will be obtained at the 
dominant wave frequency. At the same time the other parameters in the controllers $h_{R i}$, $i \in\{1,2\}$, must be chosen such that the denominator of $\delta / \psi_{H}$ does not become too small.

In the case study, Section 7, the wave filtering properties of the two controllers will be investigated further.

\section{Stability properties}

In addition to good wave filtering properties, it is important for an autopilot to have good stability properties. An analysis and discussion of stability properties of the two controllers are therefore provided in this section. We will first focus on passivity properties since this is an attractive feature for control systems. This is because the physical plant $\delta \rightarrow \dot{\psi}_{L}$ is passive, and therefore a controller with appropriate passivity properties will ensure robust stability of the closed loop system (Vidyasagar, 1993, Desoer and Vidyasagar, 1975). We will also study the asymptotic stability properties of the systems. In this section, we disregard the effect of the wave disturbances in the analysis. This means that $\psi=\psi_{L}$.

First, we prove that the passive output feedback controller in Paulsen et al. (1994) is indeed passive. We only consider the controller (7)-(9) with one virtual mass, that is $n=1$. An extension of the proof to the more general controller is trivial. Define a positive definite function as

$$
W(t)=m_{1} \dot{\psi}_{1}^{2}+k_{P 0}\left(\psi-\psi_{0}\right)^{2}+k_{P 1}\left(\psi_{0}-\psi_{1}\right)^{2}+k_{P 2} \psi_{1}^{2}
$$

Differentiating $W(t)$ with respect to time and inserting (7)-(9) yield

$$
\dot{W}(t)=-\dot{\psi} k_{P 0}\left(\psi_{0}-\psi\right)-k_{D 1}\left(\dot{\psi}_{1}-\dot{\psi}_{0}\right)^{2}-k_{D 2} \dot{\psi}_{1}^{2} \leqslant-\dot{\psi} k_{P 0}\left(\psi_{0}-\psi\right)
$$

Integrating (19) yields

$$
W(T)-W(0) \leqslant \int_{0}^{T}-\dot{\psi} k_{P 0}\left(\psi_{0}-\psi\right) \mathrm{d} t
$$

which finally gives

$$
\left\langle\dot{\psi},-k_{P 0}\left(\psi_{0}-\psi\right)\right\rangle_{T}=\langle\dot{\psi},-\delta\rangle_{T} \geqslant-W(0)
$$

which implies that $\dot{\psi} \mapsto-\delta$ is passive (Desoer and Vidyasagar, 1975). This means that the phase lag of the transfer function from $\dot{\psi}$ to $-\delta$ is less than or equal to $90^{\circ}$. For the ship and controller, this result implies that that the phase lag of the loop transfer function, $h_{R 1}(j \omega) / h_{S}(j \omega)$ will be limited by

$$
\left|\angle \frac{h_{R 1}(j \omega)}{h_{S}(j \omega)}\right|<180^{\circ}
$$

Asymptotic stability of the total system with ship and passive controller can also be proven (Paulsen et al. 1994). From this result we can conclude that the passive output feedback controller is robust with regards to stability.

The passivity properties for the general observer based controller $\delta / \psi(s)=h_{R 2}(s)$ are more complicated to investigate. However, since the relative degree of $h_{R 2}(s)$ is zero, it seems possible that the observer based controller is passive from $\dot{\psi}$ to $-\delta$ for some sets of controller parameters. In fact, design of passive LQG-controllers for passive plants is discussed in Lozano-Leal and Joshi (1988), where sufficient conditions for selecting the weighting and noise covariance matrices are given which ensure that the LQG-controller becomes passive. It should be noted that this controller will not be 
optimal with respect to the actual noise covariance matrices since the covariance matrices are used merely as controller design matrices and have no statistical meaning. In the numerical example of the present paper, the passivity properties of the observer based controller are investigated further.

Conditions for asymptotic stability are complicated to determine by studying $h_{R 2}(s)$. A design method that ensures stability of the closed loop system, is LQG-control. However, without certain restrictions on the weighting and covariance matrices, as discussed above, no stability margins are guaranteed. In general, stability in this case will be dependent on knowledge of the actual parameters of the ship.

\section{Case study: ship autopilot}

For the numerical example, we consider the system consisting of the ship model in (1) and (2), the wave model in (6), and the measurement equation (5).

The model parameters used in this example is adapted from Fossen (1993). In this reference the 'M/S Nornews Express' is described by the following set of parameters

$$
m^{*}=82.86 \quad d^{*}=2.86
$$

The length of the ship is $110 \mathrm{~m}$ and the width is $17.5 \mathrm{~m}$. The wave parameters are chosen as

$$
\omega_{n}=0.5 \quad \zeta=0.1 \quad K_{w}=0.01
$$

The parameters for the passive output feedback controller with one virtual mass, $n=1$, are chosen as

$$
\begin{aligned}
m_{1} & =0 \cdot 2 m & \zeta_{p} & =0.0001 \\
k_{D 2} & =2 \zeta_{p} \omega_{n} m_{1} & k_{P 2} & =m_{1} \omega_{n}^{2} \\
k_{D 1} & =k_{D 2} \cdot 10000 & k_{P 1} & =k_{D 1} \cdot 0 \cdot 3 \\
k_{P 0} & =k_{P 1} \cdot 4 & &
\end{aligned}
$$

The parameters for the observer based controller are chosen to be

$$
\begin{array}{ll}
k_{p}=2.033 & k_{d}=1.200 \\
k_{1}=k_{P 0} / k_{d} & k_{2}=15.029 \\
k_{3}=-10 & k_{4}=-k_{3} 2 \zeta_{0} \omega_{n} \\
\zeta_{0}=0.0001 &
\end{array}
$$

Moreover, $m=m^{*}$ and $d=d^{*}$. The parameters are chosen such that the low frequency and high frequency characteristics of the controllers are similar. Otherwise the parameters are chosen such that the noise influence on the rudder is reasonably small and the bandwidth of the loop transfer function is about $0.15 \mathrm{rad} / \mathrm{s}$.

The eigenvalues of the ship with a passive output feedback controller are

$$
\begin{aligned}
& -0.406 \pm 0.961 \\
& -0.031 \pm 0.155 \\
& -0.660
\end{aligned}
$$




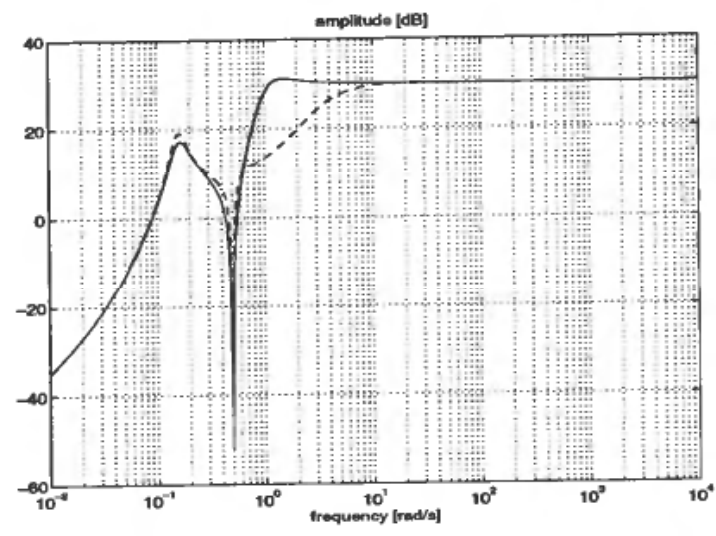

Figure 2. Amplitudes $\left|\delta / \psi_{H}(s)\right|$ in $\mathrm{dB}$ as a function of frequency. Notice that wave disturbances are suppressed around $\omega_{n}=0.5 \mathrm{rad} / \mathrm{s}$ (notch effect). Passive controller: solid line. Observer based controller: dashed line.


Figure 3. Amplitude in $\mathrm{dB}$ and phase in degrees of the loop transfer functions $h_{R i} / h_{s}$ as a function of frequency. Passive controller: solid line. Observer based controller: dashed line.

and the eigenvalues of the ship with an observer based controller are

$$
\begin{aligned}
& -3.219 \pm 1.946 \\
& -0.084 \pm 0.518 \\
& -0.0245 \pm 0.155
\end{aligned}
$$

\subsection{Frequency analysis}

The amplitudes of the transfer functions, $\delta / \psi_{H}(s)$, are shown in Fig. 2. Notch effect is obtained at the dominant wave frequency for both controllers, but the observer based controller has better damping for frequencies just above the dominant wave frequency.

In Fig. 3 the amplitudes of the loop transfer functions $h_{R i} / h_{s}$ are shown. Notice that the observer based controller is not passive from $\dot{\psi}$ to $-\delta$ since the phase of the loop transfer function $h_{R 2} / h_{s}$ is below $-180^{\circ}$ for some frequencies. Otherwise, the characteristics of the loop transfer functions are quite similar for the two systems. They 

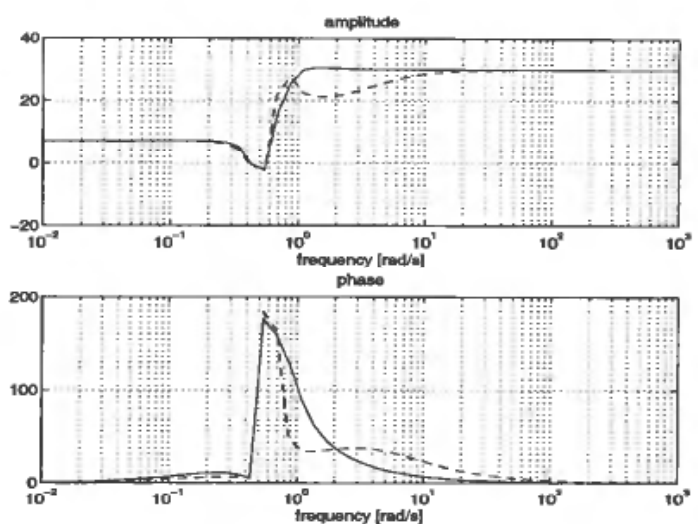

Figure 4. Amplitude in $\mathrm{dB}$ and phase in degrees of the controller transfer functions- $\delta / \psi$ as a function of frequency.

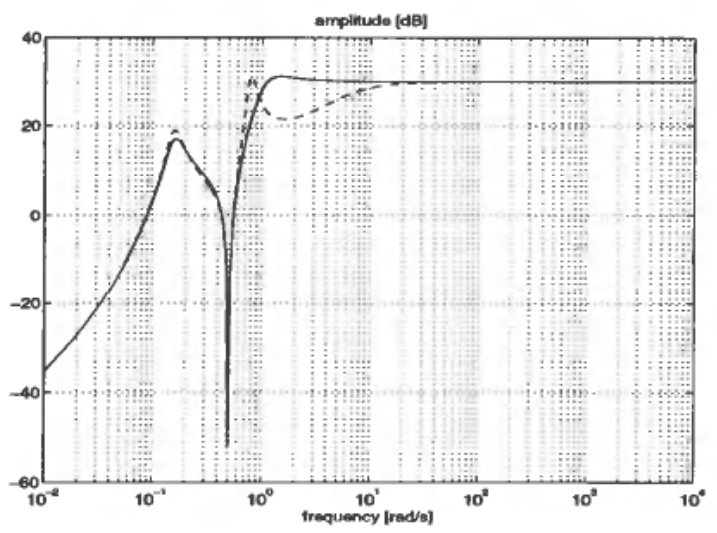

Figure 5. Amplitudes $\left|\delta / \psi_{H}(s)\right|$ in $\mathrm{dB}$ as a function of frequency.

have the same bandwidth at $0 \cdot 15 \mathrm{rad} / \mathrm{s}$. If the parameter $k_{2}$ in the observer based controller is changed to $k_{2}=2.029$, the amplitude and phase of $-\delta / \psi$ is as shown in Fig. 4. Since the phase is above 0 deg for all frequencies, this implies that for these set of parameters, the observer based controller is also passive from $\dot{\psi}$ to $-\delta$. The wave filtering characteristics for these parameters are given in Fig. 5. Comparing Fig. 2 and 5 indicates that better stability properties comes at the cost of poorer wave filtering.

Note that. for high frequencies, the noise is amplified by a factor equal to $k_{P 0}$. This may be very undesirable in the presence of high frequency noise, like measurement noise. A low pass filter can be added to the controller to remove this noise influence from the rudder. In this case, none of the controllers will be passive. However, by choosing the low pass filter wisely, stability can still be ensured.

\subsection{Simulation study}

In the simulation study a sampling frequency of $10 \mathrm{~Hz}$ is used. The Runge-Kutta fourth-order method is implemented for numerical integration.

The wave disturbance $\psi_{H}$ given by (6) and shown in Fig. 6, is acting on the output of the ship. We investigate the response of the ship with three different controllers, a 


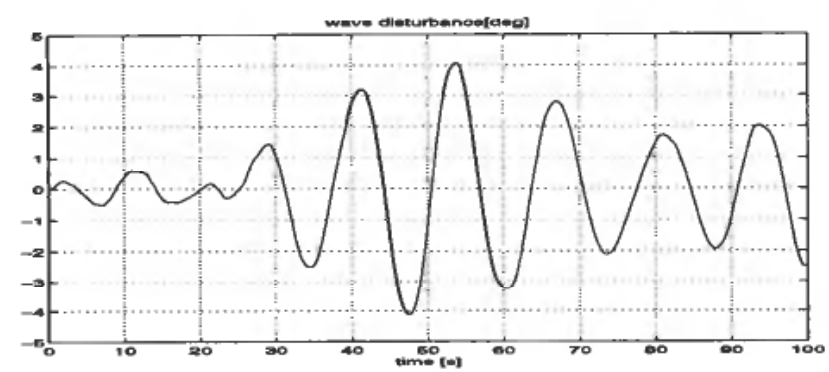

Figure 6. Wave disturbance $\psi_{H}$.

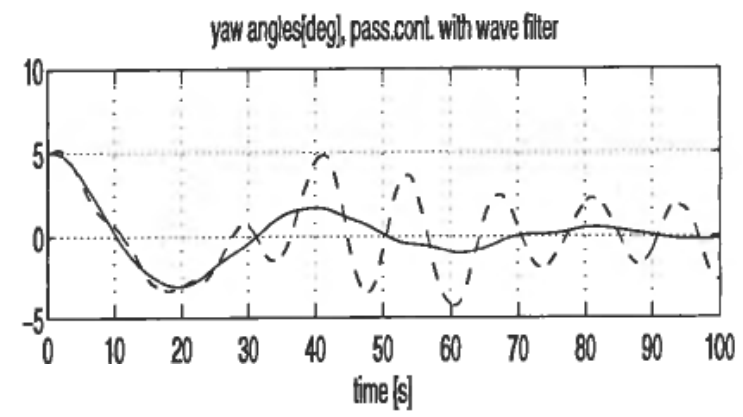

yaw anglesideg), obs.based cont.



yaw anglesideg], pass.cont, without wave fitter



Figure 7. Yaw angles, $\psi_{L}$ (solid line) and $\psi$ (dashed line) as functions of time.

passive output feedback controller (7)-(9) with wave filter $(n=1)$, and observer based controller, (12), (13), and (16), and a passive output feedback controller (7)-(9) without wave filter $(n=0)$. The parameters are chosen as in the frequency analysis.

The low frequency yaw angles $\psi_{L}$ (solid line) and measured yaw angles $\psi$ (dashed line) are presented in Fig. 7. The rudder angles are presented in Fig. 8. It clearly shows that wave filtering is obtained for both the passive output feedback controller 


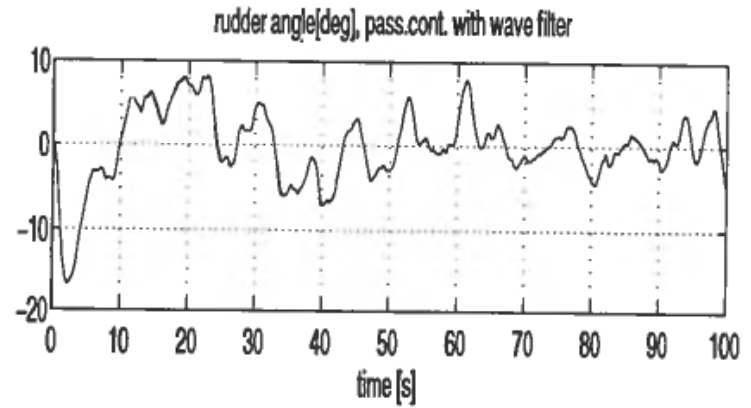

nudder angieideg], obs.based cont.

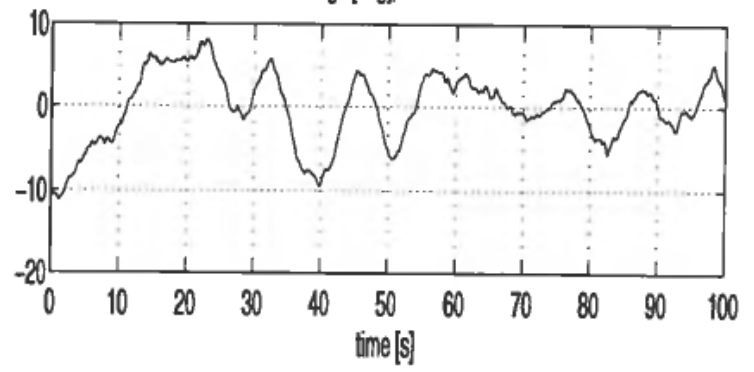

nudder angle[deg], pass.cont. without wave fitter

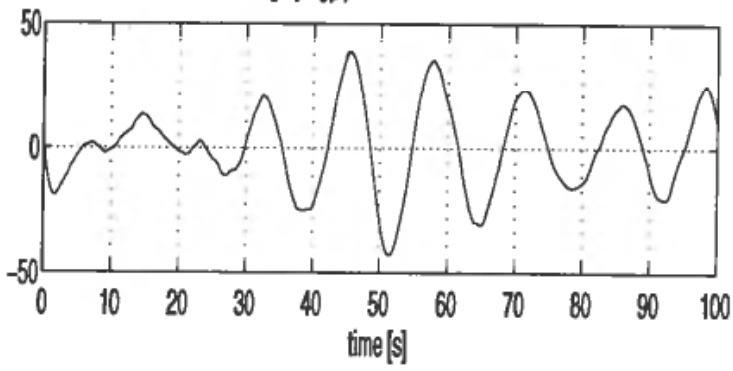

Figure 8. Rudder angles, $\sigma$ as functions of time.

$(n=1)$ and the observer based controller. No significant difference in wave filtering performance can be observed for these two controllers. In comparison, the passive controller without wave filter $(n=0)$ has poor wave filtering properties, as expected.

\section{Concluding remarks}

A comparison between an observer based controller, for instance an LQG-controller, and a passive output feedback controller with special attention to wave filtering and stability properties is given.

The two controllers are shown to have the same relative degree, but the passive controller is of order three and the observer based controller of order four. The observer based method is of highest order, and thus, a higher degree of freedom is obtained in the design of this controller. However, the design complexity increases similarly.

From the analysis it is not easy to determine which method that has the best wave filtering properties. However, our numerical example indicates that the observer based controller has stronger damping at some frequencies just above the dominant wave frequency than the passive controller. This indicates that the observer based controller may be less sensitive to some changes in the dominant wave frequency. 
Passivity of the output feedback controller is easy to establish. The controller is passive as long as the control parameters are chosen positive, and stability is ensured independently of the ship's actual parameters. It is clear that the observer based controller is not passive for all choices of controller parameters. In the frequency analysis two different choices of parameters for the observer based method are implemented, and in one case the controller is passive. Thorough considerations are necessary when choosing the control parameters for the observer based method to ensure stability.

The passive output feedback controller in this paper can be extended for MIMO marine vehicles with nonlinear dynamics. Global asymptotic stability in the sense of Lyaopunov is proven also in this case (Paulsen et al. 1994). The stability properties for the observer based method for nonlinear vehicles are more difficult to establish.

The simulation study comparing the two controllers and a controller without wave filter, showed that good wave filtering properties were obtained for both the passive output feedback controller and the observer based controller.

From the results in this paper it is not clear that one of the controllers is to be preferred to the other in all cases. However, this paper points out some of the differences of the two approaches.

\section{REFERENCES}

BalChen. J. G., Jensen, N. A. and SAelid, S. (1980). Dynamic positioning of floating vessels based on Kalman filtering and optimal control. In: Proceedings of the 19th IEEE Conference on Decision and Control. New York, USA. pp. 852-864.

Balchen, J. G., Jenssen, N. A. and SAelid, S. (1976). Dynamic positioning using Kalman filtering and optimal control theory. In: IFAC/IFIP Symp. on Automation in Offshore Oil Field Operation. Amsterdam, Netherlands. pp. 183-186.

Desoer, C. A. and Vidyasagar, M., (1975). Feedback Systems: Input-Output Properties (Academic Press, New York, USA).

Fossen, T. I. (1993). Autopilot design for nonlinear ship steering. In: Proceedings of the 10th International Ship Control Systems Symposium (SCSS'93). Ottawa, Canada. pp. 2.271-2.285.

Fossen, T. I. (1994). Guidance ard Control of Ocean Vehicles (John Wiley \& Sons Ltd, Chichester, England).

GRIMBLE, M. J. (1978). Relationship betwcen Kalman and notch filters used in dynamic ship positioning systems. Electronics Letters 14(13), pp. 399-400.

Grimble, M. J., PATtON, R. J. and WisE, D. A. (1980a). The use of Kalman filtering techniques in dynamic ship positioning control systems using stochastic optimal control theory. Optim. Control Appl. Methods, No. 1, pp. 167-202.

Grimble, M. J., PATton, R. J. and WISE, D. A. (1980b). Use of Kalman filtering techniques in dynamic ship positioning systems. In: IEE Proceedings, 127, D, No. 3. pp. 93-102.

HiLl, D. J. and MoYlan, P. J. (1977). Stability results for non-linear feedback systems. Automatica, 13, pp. 377-382.

JOHANNESSEN, E. A. and EGELAND, O. (1993). Dynamic positioning with wave filtering using a passive controller structure. In: Proc. of the 32nd IEEE Conf. on Decision and Control. San Antonio, USA. pp. 1903-1908.

Lozano-Leal, R. and Joshi, S. M. (1988). On the Design of Dissipative LQG-Type Controllers. In: Proceedings of the 27th Conference on Decision and Control. Austin, USA. pp. 1645-1646.

Nomoto, K., TAGUCHI, T., Honda, K. and Hirano, S. (1957). On the Steering Qualities of Ships. Technical report. Int. Shipbuilding Progress, Vol. 4.

Paulsen, M. J., Egeland, O. and Fossen, T. I. (1994). An output feedback controller with wave filter for marine vehicles. In: Proceedings of the 13th American Control Conference. Baltimore, USA. pp. 2202-2207. 
SÆLID, S., Jenssen, N. A. and Bai.CHEN, J. G. (1983b). Design and analysis of a dynamic positioning system based on Kalman filtering and optimal control. IEEE Transactions on Automatic Control, AC-28(3), pp. 331-339.

SARGENT, J. S. and Cowgill, P. N. (1976). Design considerations for dynamically positioned utility vessels. In: Proceedings of the 8th Off-shore Technology Conference. Dallas, USA.

SCHEI, T. S. (1995). Wave disturbance filtering in dynamic positioning systems. In: Proceedings of the IFAC workshop on Control Applications in Marine Systems, CAMS '95. Trondheim, Norway.

Vidyasagar, M. (1993). Nonlinear Systems Analysis-2nd edition (Prentice-Hall, Englewood Cliffs, NJ). 